\title{
Evaluación del efecto despigmentante en los dientes del extracto hidroalcohólico de Coffea arabica l. en pasta dental
}

\author{
Evaluation of the depigmenting effect on teeth of the hydroalcoholic extract of Coffea arabical-toothpaste
}

Carlos Alejandro Bell Cortez ${ }^{1, a, b, c}$, Diana Esmeralda Andamayo Flores ${ }^{1, a, b, c,}$, Diana Esmeralda Castillo Andamayo ${ }^{\text {2,a,ce, }}$, Renee Soledad Orrego Cabanilas ${ }^{1, a, d, f}$, Martha Raquel Valderrama Sueldo ${ }^{1, \mathrm{a}, \mathrm{b}, \mathrm{d}}$

\section{RESUMEN}

Objetivos: Determinar el efecto despigmentante del extracto hidroalcohólico de Coffea arabica L. en pasta dental. Material y Métodos: Tipo experimental: causa - efecto, prospectivo, transversal; método científico, diseño experimental. Resultados: En el estudio realizado al extracto hidroalcoholico de la Coffea arabica L, se detectaron la presencia de metabolitos secundarios como: alcaloides, tanino, compuestos fenolicos, flavonoides y azucares reductores. La pasta dental, formulada a base del extracto hidroalcohólico de Coffea arabica L. (café) tiene efecto despigmenntante en los dientes, además de aumentar el estado de vigilia. De acuerdo con la escala calificadora de Balbuena Gonzáles y Chapano Jairo, el 25,00 \% fue evaluado como excelente, el 47,50 \% como bueno, el 15,00 $\%$ como regular y el 12,50 \% como nulo. El estudio de estabilidad acelerado practicado a la pasta dental, demostró que ésta es estable y perfectamente compatible con el extracto hidroalcohólico al $2 \%$, no evidenciándose cambios organolépticos ni fisicoquímicos, por lo que se puede afirmar que tendría un tiempo de vida media de tres años. La valoración estética llevada a cabo con 50 voluntarios dejó la evidencia que el extracto hidroalcohólico Coffea arabica L. (café), tiene efecto en el proceso de despigmentación de los dientes y de aumentar el estado de vigilia de los usuarios debido probablemente a la absorción de la cafeína a través de las encías. Conclusiones: Los resultados obtenidos son óptimos cuando la pasta dental contiene $2 \%$ del extracto hidroalcohólico de Coffea arabica L. (café), teniendo un efecto despigmentante en los dientes, se observó que no hubo molestias durante el uso, el sabor y olor fueron bien tolerados y hubo una evolucion favorable.

PALABRAS CLAVES: Coffea Arabica L., despigmentante, extracto hidroalcohólico.

\section{SUMMARY}

Objectives: To determine the depigmenting effect of Coffea arabica L. hydroalcoholic extract in toothpaste. Material and Methods: Experimental type: cause - effect, prospective, transversal; scientific method, experimental design. Results: In the study carried out on the hydroalcoholic extract of coffea arabica $L$, the presence of secondary metabolites such as alkaloids, tannins, phenolic compounds, flavonoids and reducing sugars were detected. What toothpaste, formulated based on the hydroalcoholic extract of Coffea arabica L. (coffee) has a depigmenting effect on the teeth, in addition to increasing the waking state.According to the rating scale of Balbuena Gonzales and Chapano Jairo, $25.00 \%$ was evaluated as excellent, $47.50 \%$ as good and $15.00 \%$ as regular and $12.50 \%$ as null. The study of accelerated stability performed on toothpaste, showed that it is stable and perfectly compatible with $2 \%$ hydroalcoholic extract, not showing organoleptic or physicochemical changes, so it can be said that it would

Universidad Privada de Huancayo Franklin Roosevelt. Huancayo, Perú.

Universidad Peruana Cayetano Heredia. Lima, Perú.

Docente; ${ }^{\mathrm{b}}$ Químico Farmacéutico; ${ }^{\mathrm{c}}$ Doctor; ${ }^{\mathrm{d}}$ Magister; ${ }^{\mathrm{e}}$ Cirujano Dentista; ${ }^{\mathrm{f}}$ Tecnólogo Médico 
have a half-life of three years. The aesthetic evaluation carried out with 50 volunteers left the evidence that the hydroalcoholic extract Coffea arabica L. (coffee), has an effect on the process of depigmentation of the teeth and increasing the waking state of the users probably due to the absorption of Caffeine through the gums. Conclusions: The results obtained are optimal when the toothpaste contains $2 \%$ of the hydroalcoholic extract of Coffea arabica L. (coffee), having a depigmenting effect on the teeth, it was observed that there were no discomforts during use, the taste and smell were good tolerated and there was a favorable evolution.

KEYWORDS: Coffea arabica L., depigmenting, hydroalcoholic extract.

\section{INTRODUCCION}

Actualmente existen muchos tratamientos blanqueantes de los dientes debido a las grandes demandas estéticas de los pacientes. Consisten en la aplicación de una sustancia sobre el diente que es capaz de aclarar el color del mismo. Existen comercialmente dentífricos blanqueantes, es importante conocer su grado de abrasividad sobre el esmalte dentario.

Alteraciones del color dental producidas por la placa de origen microbiano son extraordinariamente frecuentes y con presentaciones clínicas de un cromatismo muy variado, desde las conocidas discoloraciones cervicales negras, que aparecen, sobre todo, en los dientes temporales, hasta las de color verde, naranja u otros colores menos frecuentes. El tabaco es una fuente importante de tinción de la placa, al igual que los colorantes alimentarios. También hay fármacos que en forma de colutorio pueden provocar tinciones como son los de clorhexidina (1).

Los productos odontológicos que se auto administra el paciente y son de venta libre se encuentran dentro del grupo OTC, es decir los dentífricos y los enjuagatorios orales. Son coadyuvantes de los métodos mecánicos del control de la placa dental. Contienen agentes quimio-terapéuticos, con diversos mecanismos de acción sobre los microorganismos que se encuentran en la placa dental, contribuyendo a la desorganización de la misma.

Actualmente, existe un gran volumen de estos productos, que hacen difícil la elección y confunden en el momento de la compra de los mismos. La Academy of General Dentistry (AGD) dice con respecto a ellos, que también existen campañas publicitarias en los diferentes medios de comunicación, que pueden resultar engañosas, destacando únicamente las propiedades beneficiosas de los mismos (2).

El café (Coffea arabica L.) es la bebida que se obtiene a partir de las semillas tostadas y molidas de los frutos de la planta del café (cafetos) y cuando se consume sin azúcar, puede considerarse una planta medicinal porque presenta muchas propiedades beneficiosas antibact erianas, antiinflamatorias y antioxidantes.

El objetivo del trabajo fue determinar el efecto despigmentante del extracto hidroalcohólico de Coffea arabica $\mathrm{L}$. en pasta dental.

\section{MATERIAL Y METODOS}

Tipo de estudio: Experimental: causa - efecto, prospectivo, transversal.

Area de estudio: Material botánico: semillas de Coffea arabica L. (café)

Grupo experimental: serán todos los pacientes con historia clínica de un centro de Salud en el Departamento y Distrito de Lima.

Poblacion de estudio: Serán 50 personas voluntarias (25 mujeres y 25 varones), entre 20 y 50 años.

Sujetos de estudio: 50 Pacientes voluntarios firmando el consentimiento informado escrito.

\section{Recoleccion de datos}

Recolección de la especie vegetal Coffea arabica $L$. (café), procedentes de la Ciudad de La Merced, Provincia de Chanchamayo, Departamento de Junín. Obtención del extracto hidroalcohólico.

Estudio fitoquimica del extracto hidroalcohólico de Coffea arabica L. (café).

Elaboración de la pasta dental con extracto hidroalcohólico de Coffea arabica L. al 2\% (café).

Medición de la actividad despigmentante de los dientes, haciendo una valoración semanal (gráfico 1).

De acuerdo con la tabla calificadora de resultados de Balbuena Gonzales y Chapano Jairo, la evaluación clínica corresponde a la clasificación como excelentes, 
buenos, regulares y nula acción despigmentante de los dientes.

\section{RESULTADOS}

De la marcha fitoquímica del extracto hidroalcohólico de Coffea arabica 1. (café), se determinó la presencia de: alcaloides, fenoles, flavonoides y azucares reductores.

Los resultados del análisis físico químico del extracto hidroalcohólico de Coffea arabica I. (café) se muestran el tabla 1.
Los resultados de la evaluación farmacotécnica de la pasta se muestran en la tabla 2.

Se ensayó la formulación que se describe, para lo cual se tuvo en cuenta que los ingredientes seleccionados sean compatibles con el extracto obtenido y que, sobre todo, sean estables en el tiempo, así como de bajo costo:

Extracto de

Café $2,00 \mathrm{~g}$ Agentes aniónicos, diluyentes y preservantes. $80,00 \mathrm{~g}$ Agua destilada estéril c.s.p. $100,00 \mathrm{~g}$

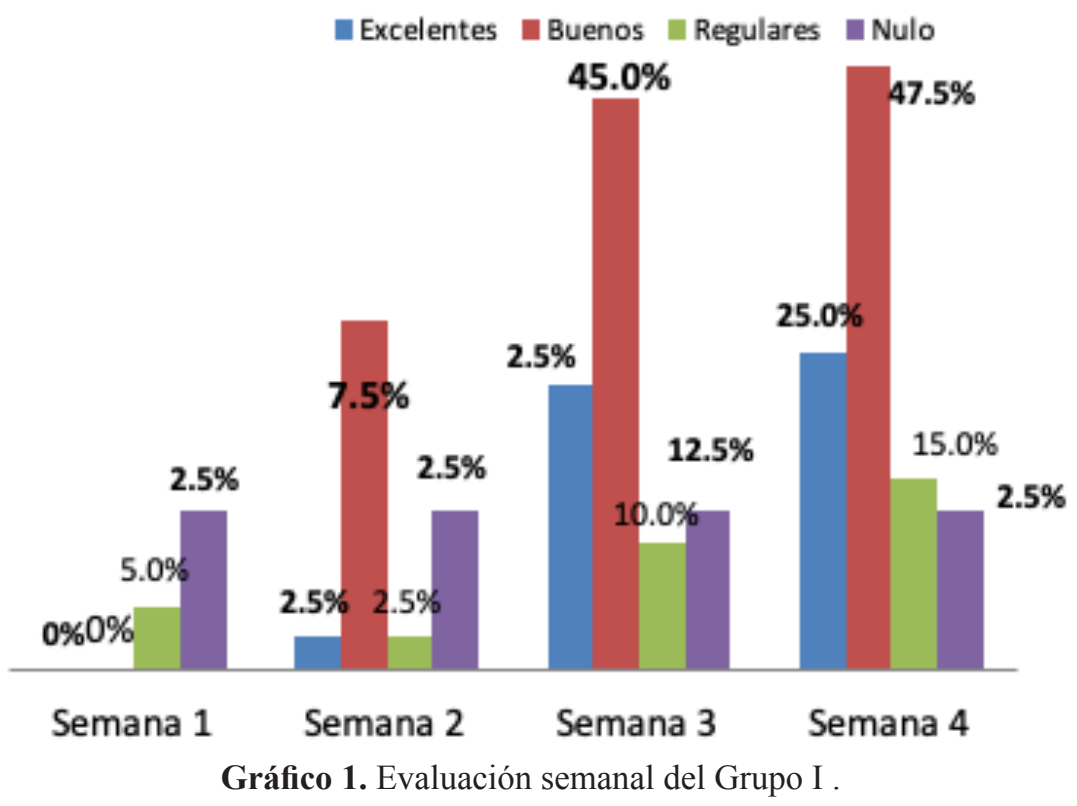

Tabla 1. Protocolo de análisis del extracto de Coffea arabica L. (café)

\begin{tabular}{llc}
\hline PRODUCTO & $:$ & Extracto de Coffea arabica L. (café). \\
\hline CANTIDAD & $:$ & $100 \mathrm{~g}$ \\
FECHA de Preparación de Extracto & $:$ & diciembre de 2018 \\
FECHA DE ANÁLISIS & $:$ & diciembre de 2018 \\
DETERMINACIONES & $:$ & RESULTADOS \\
A) FÍSICAS: & $:$ & \\
ASPECTO & $:$ & Masa blanda \\
COLOR & $:$ & Marrón claro \\
SABOR & $:$ & Amargo \\
OLOR & $:$ & Sui géneris. \\
pH (Solución alcohólica $\left.50 \%, 25^{\circ} \mathrm{C}\right)$ & 4,85 \\
B) QUIIMICAS: & & $1,96 \%$ \\
CENIZAS TOTALES & $:$ &
\end{tabular}


${ }^{\circ}$ Con el fin de determinar la estabilidad del preparado y controlar sus posibles alteraciones, se efectuaron ensayos de estabilidad acelerada, sometiendo la formulación a diferentes temperaturas (tabla 3).
Para realizar esta prueba, se procedió a evaluar cada quince días, hasta completar tres meses, los siguientes parámetros: aspecto, consistencia, color, olor y $\mathrm{pH}$ (tabla 4).

Tabla 2 Ficha técnica de Estabilidad del producto terminado.

\begin{tabular}{lll}
\hline PRODUCTO & $:$ Extracto de coffea arabica $L$. (café) \\
\hline FORMA FARMACÉUTICA & $:$ Pasta \\
PRESENTACIÓN & $:$ Tubo colapsible x $75 \mathrm{~mL}$ \\
N $^{\circ}$ DE LOTE & $:$ Piloto $N^{\circ} 001$ \\
FECHA FAB. & $:$ Febrero 2019 \\
FECHA VENC. & $:$ Febrero 2022 \\
\hline
\end{tabular}

Tabla 3. Resultados de ficha técnica de estabilidad.

\begin{tabular}{llllll}
\hline \multirow{2}{*}{ DETERMINACIONES } & \multirow{2}{*}{ ESPECIFICACIONES } & \multicolumn{4}{c}{ RESULTADOS } \\
\cline { 3 - 6 } ASPECTO & Pasta homogénea & Cumple & Cumple & Cumple & Cumple \\
COLOR & Marrón & Cumple & Cumple & Cumple & Cumple \\
OLOR & A café & Cumple & Cumple & Cumple & Cumple \\
VISCOSIDAD & $100000-150000 \mathrm{cps}$ & 140000 & 130000 & 130000 & 130000 \\
pH & $5,5-7,0$ & 6,58 & 6,72 & 6,75 & 6,70 \\
RECUENTO MICROBIANO & & & & & \\
AEROBIOS MESOFILOS & Menos de $100 \mathrm{ufc} / \mathrm{g}$ & Conforme & Conforme & Conforme & Conforme \\
RECUENTO DE HONGOS & Menos de $10 \mathrm{ufc} / \mathrm{g}$ & Conforme & Conforme & Conforme & Conforme \\
RECUENTO DE LEVADURAS & Menos de $10 \mathrm{ufc} / \mathrm{g}$ & Conforme & Conforme & Conforme & Conforme \\
ESCHERICHIA COLI & Ausente en $1 \mathrm{~g}$ & Conforme & Conforme & Conforme & Conforme \\
SALMONELLA SPp & Ausente en $1 \mathrm{~g}$ & Conforme & Conforme & Conforme & Conforme \\
STAPHYLOCOCCUS AUREUS & Ausente en $1 \mathrm{~g}$ & Conforme & Conforme & Conforme & Conforme \\
PSEUDOMONAS AERUGINOSA & Ausente en $1 \mathrm{~g}$ & Conforme & Conforme & Conforme & Conforme \\
\hline
\end{tabular}

Tabla 4. Valoración por semanas.

\begin{tabular}{|c|c|c|c|c|c|c|c|c|c|c|c|c|c|c|c|c|}
\hline & \multicolumn{4}{|c|}{$1^{\circ}$ Semana } & \multicolumn{4}{|c|}{$2^{\circ}$ Semana } & \multicolumn{4}{|c|}{$3^{\circ}$ Semana } & \multicolumn{4}{|c|}{$4^{\circ}$ Semana } \\
\hline & \multicolumn{2}{|c|}{ GRUPO I } & \multicolumn{2}{|c|}{ GRUPO II } & \multicolumn{2}{|c|}{ GRUPO I } & \multicolumn{2}{|c|}{ GRUPO II } & \multicolumn{2}{|c|}{ GRUPO I } & \multicolumn{2}{|c|}{ GRUPO II } & \multicolumn{2}{|c|}{ GRUPO I } & \multicolumn{2}{|c|}{ GRUPO II } \\
\hline & n & $\%$ & $\mathbf{n}$ & $\%$ & $\mathbf{n}$ & $\%$ & n & $\%$ & $\mathbf{n}$ & $\%$ & $\mathbf{n}$ & $\%$ & $\mathbf{n}$ & $\%$ & $\mathbf{n}$ & $\%$ \\
\hline Excelentes & - & - & - & - & 1 & 2,50 & - & - & 9 & 22,50 & - & - & 10 & 25,00 & - & - \\
\hline Buenos & - & - & - & - & 11 & 27,50 & - & - & 18 & 45,00 & - & - & 19 & 47,50 & - & - \\
\hline Regulares & 2 & 5,00 & - & - & 1 & 2,50 & - & - & 4 & 10,00 & - & - & 6 & 15,00 & - & - \\
\hline Nulo & 5 & 12,50 & 10 & 100,0 & 5 & 12,50 & 10 & 100,0 & 5 & 12,50 & 10 & 100,0 & 5 & 12,50 & 10 & 100,0 \\
\hline Total & 7 & 17,50 & 10 & 100,0 & 18 & 45,00 & 10 & 100,0 & 36 & 90,0 & 10 & 100,0 & 40 & 100,0 & 10 & 100,0 \\
\hline
\end{tabular}


Tabla 5. Evaluacion clinica.

\begin{tabular}{llc}
\hline CALIFICACIÓN & No CASOS & \% \\
\hline $\begin{array}{l}\text { EXCELENTES } \\
\text { (Reduccion entre } 80-100 \%)\end{array}$ & 10 & 25,0 \\
$\begin{array}{l}\text { BUENOS } \\
\text { (Reduccion entre } 60-80 \%)\end{array}$ & 19 & 47,5 \\
$\begin{array}{l}\text { REGULARES } \\
\text { (Reduccion entre 40-60\%) }\end{array}$ & 6 & 15,0 \\
$\begin{array}{l}\text { NULO } \\
\text { (Menor de 40\%) }\end{array}$ & 5 & 12,5 \\
$\begin{array}{l}\text { Sub -TOTAL } \\
\text { "GRUPO CONTROL" }\end{array}$ & 40 & 100 \\
TOTALES & 10 & \\
\hline
\end{tabular}

De acuerdo con la tabla calificadora de resultados de Balbuena Gonzáles y Chapano Jairo, la evaluación clínica corresponde a la clasificación que se señala en la tabla 5 .

\section{DISCUSIÓN}

En el presente estudio de investigación se evaluó el efecto despigmentante en los dientes del extracto hidroalcohólico de Coffea arabica L. (café) en pasta dental, los resultados de esta investigación se redactan en base al cumplimiento de los objetivos y se encuentra que:

En el estudio realizado durante los 12 meses se determinaron la presencia de metabolitos secundarios como: alcaloides, tanino, compuestos fenolicos, flavonoides y azúcares reductores, lo que se corrobora con el trabajo de Mesa et al., quienes encuentran como resultado que el café ademas de contener la cafeína, tiene compuestos fenólicos y melanoidinas, efectos beneficiosos para la salud bucal y general de la población (3).

El material botánico utilizado fueron las semillas de Coffea arabica L. (café], procedente de La Merced capital del Distrito de la Provincia de Chanchamayo, Departamento de Junin, Perú a 751 msnm, a esta altura el cafeto se desarrolla muy bien y teniendo las propiedades químicas que permiten usarlo adecuadamente; lo que discrepa con el trabajo de Palomino et al., el cultivo de éste con un clima particular por su humedad y temperaturas medias máximas y mínimas (en relación a otras zonas productoras de café), altitud (entre 1000 y $1500 \mathrm{msnm})(4)$.
Al determinar el efecto despigmentante del extracto hidroalcohólico de coffea arabica $L$. en pasta dental se encontraron los siguientes resultados; excelentes resultados con un $25 \%$, buenos resultados con un $47,5 \%$, regulares resultados en un $15 \%$ y nulo resultado en un $12,5 \%$; hoy en la actualidad se están realizando investigaciones con plantas medicinales que tienen carácterísticas farmacológicas lo que sustenta el estudio de Borja, quien encuentra que los resultados al finalizar el estudio no son estadisticamente significativos (5).

\section{CONCLUSIONES}

En el estudio realizado al extracto hidroalcohólico de la coffea arabica $L$, se determinó la presencia de metabolitos secundarios como: alcaloides, tanino, compuestos fenólicos, flavonoides y azúcares reductores.

El estudio de estabilidad acelerado practicado a la pasta dental, demostró queésta es establey perfectamente compatible con el extracto hidroalcohólico al $2 \%$, no evidenciándose cambios organolépticos ni fisicoquímicos, por lo que se puede afirmar que tendría un tiempo de vida media de tres años.

Los resultados obtenidos son óptimos cuando la pasta dental contiene $2 \%$ del extracto hidroalcohólico de Coffea arabica L. (café), teniendo un efecto despigmentante en los dientes, se observó que no hubo molestias durante el uso, el sabor y olor fueron bien tolerados y hubo una evolución favorable.

El extracto hidroalcohólico de Coffea arabica L. (café) usado en forma de pasta dental, mostró tener efecto despigmentante en los dientes.

\section{Resultados de la Evaluación clinica de la Pasta dental con extracto hidroalcohólico de coffea arabica $L$.}

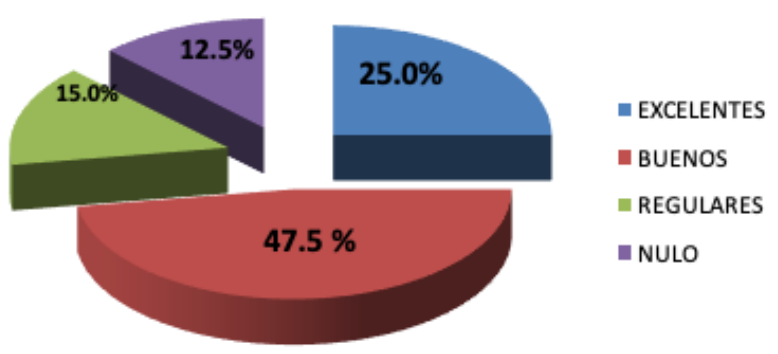

Gráfico 2. Resultados de la Evaluación Clínica 
De acuerdo con la escala calificadora de Balbuena Gonzáles y Chapano Jairo, el 25,00 \% fue evaluado como excelente, el $47,50 \%$ como bueno y el $15,00 \%$ como regular y el $12,50 \%$ como nulo (gráfico 2 ).

Por ser un producto natural, el extracto hidroalcohólico de Coffea arabica L. (café) se convierte en una alternativa para el proceso de despigmentación de los dientes.

El uso de la pasta dental formulada con extracto hidroalcohólico de Coffea arabica L. (café) mostró tener efecto en el aumento del estado de vigilia.

\section{REFERENCIAS BIBLIOGRAFICAS}

1. Galindo C, Romo C HM. Técnicas de ayuda odontológica y estomatológica. Madrid: Macmillan Iberia; 2009.
2. Costa S, Ballesteros J. Evolución de la farmacoterapeútica hacoa la aplicación raciobnal de los medicamentos. Actas Odontol. 2007;4(2):32-39.

3. Mesa N, Medrano J, Martínez M , Grave M. Efecto anticariogénico del café. Correo Científico Médico. 2017;21(3):888-98.

4. Palomino C, López C, Espejo R, Mansilla R, Quispe J. Evaluación de la diversidad genética del café Coffea arabica L en Villa Rica Perú. Ecol Apl. 2014;13(2):129-34.

5. Borja F. Eficacia de la cáscara de plátano como agente blanqueador dental en piezas premolares in vitro. Tesis de Grado. Arequipa, Perú: Universidad Alas Peruanas.
Recibido: 12/09/2018

Aceptado: 02/11/2019 